\title{
Perioperative Care of Patients With Obstructive Sleep Apnea
}

\author{
Roop Kaw, MD \\ Bhargavi Gali, $M D$ \\ Nancy A. Collop, MD
}

\author{
Corresponding author \\ Roop Kaw, MD \\ Department of Hospital Medicine and Outcomes Research, Anesthesiology \\ Cleveland Clinic, 9500 Euclid Avenue \\ Cleveland, OH 44195, USA. \\ E-mail: kawr@ccf.org
}

\section{Bhargavi Gali, MD}

Department of Anesthesiology

Mayo Clinic, 200 First Street SW

Rochester MN 55905, USA.

E-mail: gali.bhargavi@mayo.edu

Nancy A Collop, MD

Division of Pulmonary and Sleep Medicine

Emory University School of Medicine

1841 Clifton Road, NE Suite 1-106

Atlanta, GA 30329, USA.

E-mail: nancy.collop @emory.edu

Opinion statement
It has been demonstrated that patients undergoing surgical procedures are at
increased risk for complications if they have obstructive sleep apnea. It is believed that
this increase in risk is related to more difficult intubations, use of ventilatory
depressant medications, and perhaps body positioning. Although identifying patients
with a preexisting diagnosis of sleep apnea is important so they can be triaged
appropriately during the perioperative period, a bigger challenge is trying to identify
patients who require a surgical procedure and may have undiagnosed sleep apnea.
Hospitals and surgical centers should have policies in place to assist in such
identification preoperatively, with a protocol on how to manage such patients
perioperatively. Such guidelines exist, but many institutions do not have such
protocols in place or fail to ensure that they are consistently followed.
The key to the perioperative management is close observation of these high-
risk patients. In ambulatory surgery populations, these patients should be observed for
an extended period before being discharged to home. In inpatient settings, the
observation can be tailored to the patient's postoperative risks based on the type of
surgery and the severity of his or her sleep apnea.
Patients undergoing bariatric surgery are at particularly high risk. These
patients have a very high prevalence of sleep apnea and comorbid conditions.
Screening this population for obstructive sleep apnea is mandatory, and a plan for
postoperative observation should be in place in all institutions performing such
surgery.


The incidence of obesity and obstructive sleep apnea (OSA) continues to rise in the Western world. In the Wisconsin Sleep Cohort Study, the percentage of obese individuals (with a BMI [body mass index] > 40) with an apnea-hypopnea index (AHI) of 15 or higher is $42 \%$ to $55 \%$ in men and $16 \%$ to $24 \%$ in women [ 1 , Class I]. Obese patients tend to have more elective surgical procedures, and early identification of OSA in the surgical patient may be important to allow for specific interventions that can improve postoperative outcomes. The problem is further hindered by the difficulty of diagnosing OSA. Clinical examination carries a diagnostic sensitivity and specificity of only $50 \%$ to $60 \%$ for sleep apnea, even when performed by experienced sleep physicians [2, Class II]. Although data to guide the perioperative management of patients with moderate to severe OSA are scarce, heightened awareness is recommended. The purpose of this review is to identify interventions and management strategies that best facilitate improved perioperative care for patients with OSA.

\section{Prevalence of OSA in surgical patients}

No epidemiologic studies have been conducted to determine the prevalence of OSA in the general surgical population. Prevalence reports vary on the basis of whether all patients were consecutively screened or a questionnaire was used. In a series of 433 patients undergoing general surgery, 18 of 41 patients suspected to have sleep apnea agreed to undergo polysomnography, and 78\% of these patients were found to have an AHI greater than 5 [3, Class II]. When the Berlin Questionnaire was used for preoperative screening, the reported prevalence of OSA among patients undergoing surgery was $24 \%$ [4, Class II]. It was notable that OSA had not been diagnosed in the majority of these individuals. Thus a significant number of patients with sleep apnea may present for surgery without receiving a prior diagnosis.

\section{Treatment}

\section{Patient history and screening questionnaires}

- Inquiry about heavy snoring, sudden awakenings with a choking sensation, and witnessed apneas by a bed partner should be a routine component of the preoperative visit. The severity of these items in the history correlates with the severity of sleep study-proven OSA [5, Class I]. It should be recognized that patients with a known diagnosis of OSA who have undergone uvulopalatopharyngoplasty and no longer snore may still have residual OSA and may warrant further evaluation.

- The Berlin Questionnaire is an 11-item self-report instrument. It has been validated in the primary care setting, with a sensitivity of 0.89 and a specificity of 0.71 , but the scoring procedure is complex.

- The American Society of Anesthesiology (ASA) checklist requires a clinician to complete the evaluation.

- A tool that is much easier to use is the STOP (S, Snore loudly; T, day-time Tiredness; O, Observed to stop breathing during sleep; P, high blood Pressure) questionnaire. The STOP-BANG questionnaire adds more variables (B, BMI>35; A, Age $>50$ years; N, Neck circumference $>40 \mathrm{~cm}$; and G, male Gender), and at a cutoff score of $\geq 3$, it has $93 \%$ sensitivity and $47 \%$ specificity for an AHI cutoff of greater than 15. For an AHI cutoff greater than 30, the STOP-BANG sensitivity is $100 \%$ and its specificity is $37 \%$ [6, Class II]. 
- Physical examination may reveal the characteristic stigmata of OSA, including a short, thick neck; nasal obstruction; tonsillar hypertrophy; narrow oropharynx; and retrognathia. Although these clinical features are typical, they are not reliable predictors of the presence or severity of OSA [7, Class III].

- Clinical suspicion for OSA may also first be recognized intraoperatively if the patient has problems with maintenance of the airway, proves difficult to intubate, or is observed postoperatively to be snoring or having obstructions. Airway obstruction out of proportion to the apparent degree of sedation can also suggest undiagnosed OSA. The degree of difficulty in visualizing the faucial pillars, the soft palate, and the base of the uvula predicts difficulty with intubation and should increase the suspicion of OSA [8, Class II]. Eastwood et al. suggest that sleep-disordered breathing should be considered in all patients with a pronounced tendency for upper airway obstruction during anesthesia or recovery from anesthesia [9, Class II].

\section{Overnight oximetry}

- Polysomnography remains the "gold standard" for diagnosing and treating OSA, but restrictions on access and practical application may limit its utility in the preoperative setting. Therefore, alternative screening tools have been tested to assess for the severity of sleep-disordered breathing preoperatively. In their study using preoperative overnight oximetry in 24 patients, Reeder et al. [10, Class II]. demonstrated "significant abnormalities of respiration" (SARs) postoperatively in all patients with preoperative SARs. SARs were defined as dips in oxygen saturation of $4 \%$ or more at least five times in 1 hour. However, the preoperative nocturnal oximetry identified only one third of those who experienced postoperative SARs. Home nocturnal oximetry has been studied as a potential screening tool, but a wide range of sensitivity and specificity for OSA has been reported $[11,12$, Class III].

- In contrast, a multicenter Japanese study [13, Class II] conducted with patients having elective surgery established that the presence of preoperative sleepdisordered breathing, as evidenced by an elevated preoperative Oxygen Desaturation Index (ODI), measured by the number of episodes of oxygen desaturation of $4 \%$ or more per hour, and witnessed apneas was positively associated with the severity of postoperative nocturnal desaturations. ODI is presumed to detect a pattern of deoxygenation most consistent with sleepdisordered breathing, and thus it has been used as a surrogate marker, rather than 
a marker for severity of OSA. In the outpatient setting, an ODI of 10 or higher has been reported to have sensitivities of $71 \%$ to $85 \%$ and specificities of $90 \%$ to 93\% [14, Class II $]$.

- In a more recent study using home nocturnal oximetry testing in patients who were preselected with clinical features of OSA during a preoperative evaluation, perioperative complications were noted in about $10 \%$ of the patients. About $88 \%$ of these complications occurred in the $57 \%$ of patients who had five or more episodes of $4 \%$ oxygen desaturation per hour. The OR, adjusted for sex and BMI, was 7.2 compared with the group having fewer than five episodes $(P=0.012)$ $[15$, Class II]. This study is limited by the fact that screening oximetry was not used in unselected preoperative patients, and technical factors such as oximetry sampling may affect the determination of the ODI.

- We do not yet know which tool is the most efficient, cost-effective, and reliable in assessing the presence and severity of OSA. Until further data have answered this question, a formal polysomnogram should be performed in high-risk patients, if it is feasible in the given clinical situation. This recommendation may be particularly important for highly suspect surgical patients such as those undergoing open Roux-en-Y gastric bypass.

\section{Decisions regarding surgical setting: ambulatory or inpatient?}

- Factors to consider when evaluating how patients with suspected OSA should be monitored postoperatively include the preclinical suspicion of the severity of OSA, the type of surgery being performed, the need for postoperative narcotics, and the clinical course in the recovery room. Surgery requiring only regional anesthesia or a limited need for postoperative narcotic analgesia should be considered for the outpatient setting. These patients can be sent home when fully conscious if they are not snoring and do not have an obstruction in the recovery room. The ASA guidelines recommend outpatient surgery for superficial surgeries using local or regional anesthesia, minor orthopedic surgery with local or regional anesthesia, and lithotripsy [16, Class III], but as they are only consensus-based, they are equivocal about ambulatory scheduling of superficial or minor orthopedic surgeries and gynecologic laparoscopy done under general anesthesia.

- Patients who are expected to have significant pain or require opioid therapy, who have severe OSA at baseline that requires continuous positive airway pressure (CPAP) therapy at home, or who have an observed obstruction or episodic desaturations that are evident in the recovery room should be considered for 
continued inpatient monitoring. A recent study by Stierer et al. [17•, Class II] reported no unplanned hospital admissions after ambulatory surgery in patients with greater than $70 \%$ propensity for OSA based on their prediction model. Increased propensity for OSA was associated with difficult intubation; intraoperative tachycardia and use of intravenous labetalol, ephedrine, or metoprolol; and increased desaturation in the postanesthesia care unit (PACU), but no need for assisted ventilation.

\section{Choosing the head position}

- Different schools of thought exist regarding the head position required for optimal upper airway stability in surgical patients with OSA. Positioning of obese patients with known or anticipated OSA should include positioning with a ramp of blankets to elevate the torso and head and achieve the "sniffing position" [18, Class II]. Upper body elevation relieves OSA by increasing the stability of the upper airway. Lateral (nonsupine) head position has been suggested by some to improve upper airway stability during sleep and also to allow for reduction of therapeutic levels of CPAP [19, Class II]. ASA guidelines recommend a semiupright position for extubation and recovery and a nonsupine postoperative position [16, Class III].

\section{Selecting sedation and analgesia}

- Alterations in consciousness from sedative medication or induction of anesthesia can exacerbate the collapsibility of the upper airway of the patient with OSA [20, Class III]. The immediate preoperative period often includes administration of sedative agents to relieve anxiety or provide analgesia. In patients with OSA, this can lead to obstruction, so sedation should always be given to the OSA patient in a supervised setting with continuous observation of the patient. Local or regional anesthesia should be considered for the surgical procedure or as an adjunct to general anesthesia [21, Class II]. These techniques may reduce concerns about upper airway collapse during procedures. In addition, the use of regional anesthesia may allow decreased use of opioids and other sedatives throughout the perioperative period.

- Anatomy associated with OSA (increased neck circumference, macroglossia, retrognathia, and maxillary constriction) can narrow the airway, making mask ventilation and intubation challenging. A high incidence of OSA has been found in patients with unexpectedly difficult intubation [22, Class II]. Preparation for induction and intubation should follow the ASA difficult-airway guidelines [23, Class IV]. Preoxygenation, performed by providing $100 \%$ oxygen via a tight- 
fitting anesthesia mask for 3 minutes, can increase the time of tolerance of apnea in case of difficulties with intubation [24, Class II]. Alternative airway devices (such as a laryngeal mask airway, videolaryngoscope, or fiberoptic scope) should be easily available as options in case intubation is more challenging than anticipated.

- Avoiding long-acting anesthetic medications may be preferred in OSA patients, as their effects may persist after surgery. Short-acting agents such as desflurane, propofol, and succinylcholine are recommended, although there is limited literature in this area. Dosing of medications should be adjusted for obese patients. For example, basing a fentanyl dose on pharmacokinetic mass will decrease the relative dose of fentanyl as total body weight increases [25, Class II].

- Many surgical procedures require the use of long-acting, nondepolarizing muscle relaxants. Full reversal of the effects of muscle relaxants is important in these patients to avoid postoperative upper airway obstruction; the reversal should be assessed with the use of a nerve stimulator before extubation [26, Class III]. Certain procedures may require special precautions with extubation. Surgery on the neck or in the patient's mouth, or procedures that require long periods of Trendelenburg positioning or large amounts of intravenous fluids, can require time and repeated reassessment before extubation. Besides confirming complete reversal of neuromuscular blockade, it is important that the patient be fully awake, hemodynamically stable, normothermic, and breathing spontaneously (with an adequate respiratory rate and tidal volume) prior to extubation.

- Assessment in the immediate postoperative period includes monitoring for evidence of obstruction and asynchrony of respiratory efforts. Providing safe postoperative analgesia in patients with OSA can be challenging. Opioids, the most commonly used class of analgesics, decrease hypoxic and hypercapnic ventilatory drive [27, Class III]. Regional blocks or catheters may be useful in decreasing the need for opioids [16, Class III], but it is important to keep in mind the risk from opioids administered intrathecally or via epidural injection [28, Class II].

- Multimodal analgesia can be used to decrease the need for opioids and thus the complications that may result in OSA patients [20, Class III]. Multimodal analgesia can include the use of nonsteroidal anti-inflammatory drugs, including 
acetaminophen, tramadol, ketamine, pregabalin, and COX-2 inhibitors.

Perioperative oxygen desaturations are more likely to occur in OSA patients receiving opioids than in those using nonopioid analgesic agents [29, Class III].

\section{Supplemental oxygen}

- Although the literature to support its use is scarce, postextubation supplemental oxygen should be used in patients with OSA until they are able to maintain their baseline oxygen saturation on room air. The ASA task force cautions that supplemental oxygen may increase the duration of apneic episodes and may hinder the detection of transient apnea, atelectasis, and hypoventilation.

\section{Monitoring}

- Guidelines to address postoperative and intensive care monitoring have been proposed by some authors, though they are based more on expert opinion than on evidence [30, Class IV]. It should be recognized that an unsupervised holding area is inappropriate for a premedicated patient with sleep apnea. The ASA guidelines recommend that continuous oximetry may be used in the critical care or step-down unit for patients with increased perioperative risk from OSA, but these guidelines do not support the need for intermittent or continuous oxygen monitoring in all patients. The guidelines are equivocal about full monitoring in the ICU setting or continuous oximetry by a dedicated observer in the patient's room or for low-risk patients no longer receiving continuous parenteral narcotics.

- Bolden et al. [29, Class III], in a study of 221 surgical patients with OSA, reported more likely postoperative hemoglobin oxygen desaturation in patients receiving intravenous opioids (OR,14; 95\% CI, 3-65; $P<0.001)$, as opposed to those receiving oral opioids (OR, $12 ; 95 \% \mathrm{CI}, 3-58 ; P<0.001)$, particularly in first 24 hours after surgery (OR, 21; 95\% CI, 6-74; $P<0.0001)$. All patients were managed per a perioperative OSA protocol as part of a patient safety initiative; $88 \%$ were admitted to a designated OSA bed on a general nursing floor and the other $12 \%$ were in a step-down or ICU setting.

- In a more recent clinical practice improvement initiative, Gali et al $[31 \bullet \bullet$, Class II] tested sleep apnea clinical score (SACS) criteria based on Flemons criteria [32, Class II], using neck circumference, history of hypertension, and reported clinical symptoms to screen for patients at high risk for oxygen desaturation after PACU discharge. An SACS of 15 or higher has been reported to predict a likelihood ratio of 5.17 and a posttest probability of $81 \%$ for OSA [31••, Class II], and hence unplanned ICU admission was much less frequent among patients 
with a low SACS $(0.5 \%)$ versus those with a high SACS $(8.8 \%$; RR, $16.9 ; 95 \%$

CI, 8.2-35.2) [33, Class III]. An ODI greater than 10 after PACU discharge was reported in $29 \%$ of patients with SACS of 15 or higher and in $44 \%$ of patients with this high SACS and recurrent PACU events, compared with 19\% in those with SACS lower than 15 and $24 \%$ in those with low SACS and recurrent PACU events $[31 \bullet \bullet$, Class II]. The patients with a high SACS and recurrent PACU events had the highest likelihood of respiratory complications (33\%), but polysomnography was not performed to confirm OSA in any of these patients.

- Based on clinical experience, patients that are considered to have high risk for OSA by prescreening and have recurrent PACU respiratory events should receive a higher level of care. The ASA recommends longer (3 hours) postoperative monitoring in OSA patients after ambulatory surgery and 7 hours prior to discharge from the facility after the last episode of airway obstruction or hypoxemia while breathing room air in an unstimulated environment [16, Class III]. The definition of higher level of care is institution-dependent, as some institutions have remotely monitored oximetry. This is continuous observation of oximetry data by a trained respiratory therapist, who can alert nurses caring for a patient when any significant changes or downward trends occur in a patient's oximetry. Alternatively, a step-down unit with a higher nurse-patient ratio should be considered in hospitals who do not have remote oximetry capability.

- The need for opioids and patient positioning should also be considered in the recovery period. If a patient shows a high risk for OSA on screening or has recurrent PACU respiratory events and requires high-dose opioids and a supine position, this patient should also be considered for remote oximetry or placement in a step-down unit.

\section{Perioperative CPAP}

- No studies have conclusively shown CPAP therapy to be beneficial in the postoperative setting, although adequate randomized controlled trials to assess its efficacy have not been performed. Routine use of CPAP in the immediate postoperative period in CPAP-naïve or previously noncompliant patients is not usual practice. However, if frequent episodes of severe obstruction occur, CPAP use should be considered [16, Class III].

- In individuals with a known diagnosis of OSA who are receiving CPAP therapy, it is thought that the perioperative use of CPAP will reduce the risk of postoperative complications [34, Class III]. Nasal CPAP therapy is highly effective at preserving airway patency during sleep, and over several weeks it can 
improve the diminished reflex responses to hypoxia and hypercapnia [35, Class III]. Hemodynamic fluctuations accompanying early episodes of respiratory obstruction in a patient with undiagnosed sleep apnea after aortic reconstructive surgery were abolished with nasal CPAP therapy [36, Class IV]. Gupta et al. found that use of home CPAP therapy in patients with an established diagnosis of OSA produced possible carryover protection, resulting in a significantly lower rate of complications even though most of these patients did not receive CPAP therapy in the hospital [37, Class III]. This effect may in part be related to upper airway stabilization, which is a residual effect of CPAP therapy following as little as 4 hours of use [38, Class II]. Treatment with CPAP significantly reduces the total duration of ST-segment depression in persons with sleep apnea [39, Class II]. In patients cardioverted for atrial fibrillation, the likelihood of recurrence of the atrial fibrillation within 12 months was double in those with untreated sleep apnea versus the patients with OSA who received CPAP therapy [40, Class II]. These data suggest that there may be some benefit to the use of CPAP in the perioperative setting for patients with suspected OSA, although this benefit remains to be proven. ASA guidelines did not reach any consensus on whether CPAP should be administered if there is evidence of apneas and desaturation, or if hypoxia persists with supplemental oxygen. Bolden et al. reported no possible prevention of oxygen desaturations with the use of CPAP [29].

- One suggested approach to surgical patients who are suspected of having OSA but are unable to undergo polysomnographic evaluation would be to empirically start administering CPAP in the perioperative setting, using self-adjusting or autoadjusting CPAP devices. This approach has not been studied and may have significant limitations on its implementation. If the patient is not familiar with CPAP therapy, technical difficulties in initiating CPAP therapy postoperatively may limit its effectiveness. In addition, titrating CPAP for the first time in the postoperative period is less likely to be successful.

\section{Perioperative management of OSA in patients undergoing bariatric surgery}

\section{Preoperative assessment}

- Recent series of patients evaluated for bariatric surgery have reported varying prevalences of OSA in these patients: O'Keeffe and Patterson [41, Class II] reported a $77 \%$ prevalence, whereas Hallowell et al. reported $91 \%$ prevalence [42, Class III]. Among all these studies, there was no correlation between BMI and severity of OSA; in fact, Hallowell et al. reported higher prevalence of OSA 
in severely obese patients (BMI, 35-39.9) than in morbidly obese patients (BMI, 40-49.9) [42, Class II]. Additionally, routine polysomnography alone prior to bariatric surgery demonstrated a $91 \%$ prevalence of OSA, compared with $58 \%$ when clinical parameters and Epworth Sleepiness Scale (ESS) scores alone were used to screen for OSA [42, Class III]. Mandatory screening by polysomnography also reduced the number of ICU stays related to respiratory complications to $9 \%$ of the ICU transfers, compared with $34 \%$ of ICU transfers in the group not screened prior to bariatric surgery [43, Class III]. These and other authors strongly recommend polysomnography for all patients undergoing bariatric surgery [41, Class II; 42, 43, Class III].

\section{Perioperative interventions to improve oxygenation}

- At present, three approaches are suggested to improve ventilation and increase oxygenation in obese patients: high tidal volume, positive end-expiratory pressure (PEEP), and the vital capacity maneuver [44, Class III].

Reverse Trendelenburg position

The reverse Trendelenburg position (RTP) is presumed to improve oxygenation in anesthetized obese patients, as well as enabling better exposure of the subdiaphragmatic region, allowing mechanical ventilation with safe levels of airway pressure [45, Class II]. It also decreases the push of abdominal contents on the diaphragm, thereby increasing functional residual capacity (FRC) [45, Class II]. Dixon et al. demonstrated that the decrease in oxygen saturation during apnea under anesthesia is slower when the patient is positioned head-up as compared with a flat position, but any advantage with regard to lung function, atelectasis, and shunting in the $25^{\circ}$ head-up position is lost when positive-pressure ventilation is commenced [46, Class II].

\section{Pre-induction and maintenance PEEP}

The prevention or reduction of atelectasis from the induction and maintenance of general anesthesia would improve arterial oxygenation. Preoxygenation with 100\% fraction of inspired oxygen $\left(\mathrm{FIO}_{2}\right)$ and PEEP at a level of $10 \mathrm{~cm} \mathrm{H}_{2} \mathrm{O}$ for 5 minutes before the induction of general anesthesia, followed by PEEP of $10 \mathrm{~cm} \mathrm{H}_{2} \mathrm{O}$ during mask ventilation and after intubation reduce atelectasis immediately after intubation, as assessed by CT scan, and improve postintubation arterial oxygenation on $100 \% \mathrm{FIO}_{2}\left(\mathrm{PAO}_{2}\right.$ of $457 \pm 130 \mathrm{~mm} \mathrm{Hg}$ vs $315 \pm 100 \mathrm{~mm} \mathrm{Hg}$ in the control group) [47, Class II]. Whether this reduction in atelectasis is maintained (and for how long) is not known. In obese patients without reflux, the improvement 
in oxygenation that can be achieved with the preinduction use of PEEP is significant and will increase the time before desaturation begins. In contrast, in normal-weight patients, PEEP neither increased the $\mathrm{paO}_{2}$ nor decreased the A-a gradient.

Practitioners should strongly consider taking advantage of the improved arterial oxygenation offered by preinduction PEEP.

Perioperative CPAP

Prophylactic use of CPAP in the first 24 hours after surgery significantly reduces the risk of the pulmonary restrictive syndrome that occurs in morbidly obese patients after gastroplasty [48, Class II]. In one study, there was a 16\% (95\% CI, 2.9\%$29.3 \%$ ) absolute risk reduction in the rate of respiratory failure in severely obese patients with the use of noninvasive ventilation applied during the first 48 hours after extubation [49, Class II]. However, it is believed that postoperative use of CPAP carries the theoretical risk of increasing the incidence of anastomotic leaks resulting from increased flow of pressurized air into the stomach and proximal anastomosis of Roux-en-Y gastric bypass (RYGB). Huerta et al. looked prospectively at 1,067 patients who underwent RYGB; 420 were confirmed to have OSA and 159 of these were on CPAP. Out of a total of 15 postoperative anastomotic leaks, only 2 occurred in patients treated with CPAP, and no relationship was identified between CPAP use and anatomic disruption of RYGB [50, Class II]. Jensen et al. reported that CPAP or bi-level positive airway pressure (BiPAP) may be safely omitted in laparoscopic RYGB patients as long they are observed in a monitored non-ICU setting and their pulmonary status is optimized by aggressive incentive spirometry and early ambulation [51, Class III]. More recently, among patients undergoing laparoscopic bariatric surgery, noninvasive positive pressure ventilation was reported to improve lung function if initiated immediately after extubation, but there are few data on how this affects outcomes [52••, Class II].

\section{Disclosure}

No potential conflicts of interest relevant to this article were reported.

\section{References and Recommended Reading}

Papers of particular interest, published recently, have been highlighted as:

- Of importance

- Of major importance

1. Young T, Peppard PE, Taheri S. Excess weight and sleep-disordered breathing. J Appl Physiol. 2005;99:1592-1599.

2. Redline S, Strohl KP. Recognition and consequences of obstructive sleep apnea hypopnea syndrome. Clin Chest Med. 1998;19:1-19.

3. Fidan H, Fidan F, Unlu M, et al. Prevalence of sleep apnoea in patients undergoing operation. Sleep Breath. 2006;10:161-165.

4. Chung F, Ward B, Ho J, et al. Preoperative identification of sleep apnea risk in elective 
surgical patients, using the Berlin questionnaire. J Clin Anesth. 2007;19:130-134.

5. Wilson K, Stoohs RA, Mulrooney TF, et al. The snoring spectrum: acoustic assessment of snoring sound intensity in 1,139 individuals undergoing polysomnography. Chest. 1999;115:762-770.

6. Chung F, Yegneswaran B, Liao P, et al. STOP questionnaire: a tool to screen patients for obstructive sleep apnea. Anesthesiology 2008; 108:812-821.

7. Esclamado RM, Glenn MG, McCulloch TM, Cummings CW. Perioperative complications and risk factors in the surgical treatment of obstructive sleep apnea syndrome. Laryngoscope. 1989;99:1125-1129.

8. Mallampati SR, Gatt SP, Gugino LD, et al. A clinical sign to predict difficult tracheal intubation: a prospective study. Can Anaesth Soc J. 1985;32:429434.

9 Eastwood PR, Szollosi I, Platt PR, Hillman DR. Comparison of upper airway collapse during general anaesthesia and sleep. Lancet. 2002;359:1207-1209.

10. Reeder MK, Goldman MD, Loh L, et al. Late postoperative nocturnal dips in oxygen saturation in patients undergoing major abdominal vascular surgery. Predictive value of pre-operative overnight pulse oximetry. Anaesthesia. 1992;47:110-115.

11. Levy P, Pepin JL, Deschaux-Blanc C, et al. Accuracy of oximetry for detection of respiratory disturbances in sleep apnea syndrome. Chest. 1996;109:395-399.

12. Whitelaw WA, Brant RF, Flemons WW. Clinical usefulness of home oximetry compared with polysomnography for assessment of sleep apnea. Am J Respir Crit Care Med. 2005;171:188-193.

13. Isono S, Sha M, Suzukawa M, et al. Preoperative nocturnal desaturations as a risk factor for late postoperative nocturnal desaturations. $\mathrm{Br} \mathrm{J}$ Anaesth. 1998;80:602-605.

14. Chiner E, Signes-Costa J, Arriero JM, et al. Nocturnal oximetry for the diagnosis of the sleep apnoea hypopnoea syndrome: a method to reduce the number of polysomnographies? Thorax. 1999;54:968-971.

15. Hwang D, Shakir N, Limann B, et al. Association of sleep-disordered breathing with postoperative complications. Chest. 2008;133:1128-1134.

16. Gross JB, Bachenberg KL, Benumof JL, et al. Practice guidelines for the perioperative management of patients with obstructive sleep apnea: a report by the American Society of Anesthesiologists Task Force on Perioperative Management of Patients with Obstructive Sleep Apnea. Anesthesiology. 2006;104:1081-1093.

17. $\quad$ Stierer TL, Wright C, George A, et al. Risk assessment of obstructive sleep apnea in a population of patients undergoing ambulatory surgery. J Clin Sleep Med. 2010;6:467-472. This recent paper looked at patients at risk for OSA presenting to a major tertiary care ambulatory surgery center and concluded that these patients can be safely discharged to an unmonitored setting postoperatively.

18. Isono S. Optimal combination of head, mandible and body positions for pharyngeal airway maintenance during perioperative period: lesson from pharyngeal closing pressures. Seminars in Anesthesia, Perioperative Medicine and Pain. 2007;26:83-93.

19. Penzel T, Moller M, Becker HF, et al. Effect of sleep position and sleep stage on the collapsibility of the upper airways in patients with sleep apnea. Sleep. 2001;24:90-95.

20. Hillman DR, Platt PR, Eastwood PR. Anesthesia, sleep, and upper airway collapsibility. Anesthesiology Clinics. 2010;28:443-455.

21. Catley DM, Thornton C, Jordan C, et al. Pronounced, episodic oxygen desaturation in the postoperative period: its association with ventilatory pattern and analgesic regimen. Anesthesiology. 1985;63:20-28.

22. Chung F, Yegneswaran B, Herrera F, et al. Patients with difficult intubation may need referral to sleep clinics. Anesth Analg. 2008;107:915-920.

23. Rosenblatt WH, Whipple J. The difficult airway algorithm of the American Society of Anesthesiologists. Anesth Analg. 2003;96:1233.

24. Baraka AS, Taha SK, Aouad MT, et al. Preoxygenation: comparison of maximal breathing and tidal volume breathing techniques.

Anesthesiology. 1999;91:612-616.

25. Shibutani K, Inchiosa MA Jr, Sawada K, Bairamian M. Accuracy of pharmacokinetic models for predicting plasma fentanyl concentrations in lean and obese surgical patients: derivation of dosing weight ("pharmacokinetic mass"). Anesthesiology. 2004;101:603-613.

26. Murphy GS, Szokol JW, Marymont JH, et al. Residual neuromuscular blockade and critical respiratory events in the postanesthesia care unit. Anesth Analg. 2008;107:130-137.

27. Weil JV, McCullough RE, Kline JS, Sodal IE. Diminished ventilatory response to hypoxia and hypercapnia after morphine in normal man. $N$ Engl J Med. 1975;292:1103-1106.

28. Bailey PL, Lu JK, Pace NL, et al. Effects of intrathecal morphine on the ventilatory response to hypoxia. N Engl J Med. 2000;343:1228-1234.

29. Bolden N, Smith CE, Auckley D, et al. Perioperative complications during use of an obstructive sleep apnea protocol following surgery and anesthesia. Anesth Analg. 2007;105:1869-1870.

30. Benumof JL. Obesity, sleep apnea, the airway and anesthesia. American Society of Anesthesiology, Annual Meeting Refresher Course. October, 2003.

31.. $\quad$ Gali B, Whalen FX, Schroeder DR, et al. Identification of patients at risk for postoperative respiratory complications using a preoperative obstructive sleep apnea screening tool and postanesthesia care assessment. Anesthesiology. 2009;110:869-877. This important study highlights the importance of using a two-phase screening tool combining a preoperative questionnaire with PACU 
monitoring to identify patients with OSA at high risk for postoperative complications.

32. Flemons WW, Whitelaw WA, Brant R, Remmers JE. Likelihood ratios for a sleep apnea clinical prediction rule. Am J Respir Crit Care Med. 1994;150:1279-1285.

33. Gali B, Whalen FX Jr, Gay PC, et al. Management plan to reduce risks in perioperative care of patients with presumed obstructive sleep apnea syndrome. J Clin Sleep Med. 2007;3:582-588.

34. Rennotte MT, Baele P, Aubert G, Rodenstein DO. Nasal continuous positive airway pressure in the perioperative management of patients with obstructive sleep apnea submitted to surgery. Chest. 1995;107:367-374.

35. Lin CC. Effect of nasal CPAP on ventilatory drive in normocapnic and hypercapnic patients with obstructive sleep apnoea syndrome. Eur Respir J. 1994;7:2005-2010.

36. Reeder MK, Goldman MD, Loh L, et al. Postoperative obstructive sleep apnoea. Haemodynamic effects of treatment with nasal CPAP. Anaesthesia. 1991;46:849-853.

37. Gupta RM, Parvizi J, Hanssen AD, Gay PC. Postoperative complications in patients with obstructive sleep apnea syndrome undergoing hip or knee replacement: a case-control study. Mayo Clin Proc. 2001;76:897-905.

38. Hers V, Liistro G, Dury M, et al. Residual effect of nCPAP applied for part of the night in patients with obstructive sleep apnoea. Eur Respir J. 1997;10:973-976.

39. Peled N, Abinader EG, Pillar G, et al. Nocturnal ischemic events in patients with obstructive sleep apnea syndrome and ischemic heart disease: effects of continuous positive air pressure treatment. J Am Coll Cardiol. 1999;34:1744-1749.

40. Kanagala R, Murali NS, Friedman PA, et al. Obstructive sleep apnea and the recurrence of atrial fibrillation. Circulation. 2003;107:2589-2594.

41. O'Keeffe T, Patterson EJ. Evidence supporting routine polysomnography before bariatric surgery. Obes Surg. 2004;14:23-26.

42. Hallowell PT, Stellato TA, Schuster M, et al. Potentially life-threatening sleep apnea is unrecognized without aggressive evaluation. Am J Surg. 2007;193:364-367.

43. Hallowell PT, Stellato TA, Petrozzi MC, et al. Eliminating respiratory intensive care unit stay after gastric bypass surgery. Surgery. 2007;142:608612 .

Online heading: Sleep Disorders (Sudhansu Chokroverty, Section Editor)
44. Perilli V, Sollazzi L, Modesti C, et al. Comparison of positive end-expiratory pressure with reverse Trendelenburg position in morbidly obese patients undergoing bariatric surgery: effects on hemodynamics and pulmonary gas exchange. Obes Surg. 2003;13:605-609.

45. Pelosi P, Ravagnan I, Giurati G, et al. Positive endexpiratory pressure improves respiratory function in obese but not in normal subjects during anesthesia and paralysis. Anesthesiology. 1999;91:1221-1231.

46. Dixon BJ, Dixon JB, Carden JR, et al. Preoxygenation is more effective in the 25 degrees head-up position than in the supine position in severely obese patients: a randomized controlled study. Anesthesiology. 2005;102:1110-1115.

47. Coussa M, Proietti S, Schnyder P, et al. Prevention of atelectasis formation during the induction of general anesthesia in morbidly obese patients. Anesth Analg. 2004;98:1491-1495.

48. Joris JL, Sottiaux TM, Chiche JD, Desaive CJ, Lamy ML. Effect of bi-level positive airway pressure (BiPAP) nasal ventilation on the postoperative pulmonary restrictive syndrome in obese patients undergoing gastroplasty. Chest. 1997;111:665-670.

49. El-Solh AA, Aquilina A, Pineda L, Dhanvantri V, Grant B, Bouquin P. Noninvasive ventilation for prevention of post-extubation respiratory failure in obese patients. Eur Respir J. 2006;28:588-595.

50. Huerta S, Deshields S, Shpiner R, et al. Safety and efficacy of postoperative continuous positive airway pressure to prevent pulmonary complications after RouX-en-Y gastric bypass. J Gastrointest Surg. 2002;6:354-358.

51. Jensen $C$, Tejirian $T$, Lewis $C$, et al. Postoperative CPAP and BiPAP use can be safely omitted after laparoscopic Roux-en-Y gastric bypass. Surg Obes Relat Dis. 2008;4:512-514.

52.• Neligan PJ, Malhotra G, Fraser M, et al. Continuous positive airway pressure via the Boussignac system immediately after extubation improves lung function in morbidly obese patients with obstructive sleep apnea undergoing laparoscopic bariatric surgery. Anesthesiology. 2009;110:878884. This important study emphasizes the importance of the Boussignac system, a portable face mask system that can deliver elevated airway pressure upon extubation and in transit to the PACU, resulting in significant improvement in lung function.

Left running head: Sleep Disorders

Right running head: Perioperative Care of Patients with Obstructive Sleep Apnea Kaw et al. 